re Sicherheitsfragen wird CIRECS Lösungen entwickeln, um kleine und mittelständische Unternehmen auf ihrem sicheren Weg in die Cloud zu unterstützen: Am Ende der Projektphase werden konkrete anwendungsbezogene Produkte, aber auch die Vermittlung des gewonnenen Wissens in Transferveranstaltungen stehen. Unternehmen können sich hinsichtlich der Reduktion von Sicherheitsrisiken beim Cloud Computing vom Fraunhofer SIT beraten lassen.

\section{Informationssicherheit im Smart Grid}

Deutsche Normungsorganisationen DIN und DKE stellen neue Sicherheitsnorm ISO/IEC TR 27019 in Berlin vor

Die deutschen Normungsorganisationen DIN und DKE Deutsche Kommission Elektrotechnik Elektronik Informationstechnik im DIN und VDE haben am 11. September 2013 in Berlin die neue internationale Norm ISO/IEC TR 27019 Vertretern aus Wirtschaft, Forschung und Verwaltung vorgestellt. Die Norm ermöglicht es den Energieversorgern, vorhandene Prozesskontrollsysteme (Process Control Systems, PCS) und SCADA-Systeme (Supervisory Control and Data Acquisition) in das meist bestehende, auf ISO/IEC 27000 basierte Informationssicherheitsmanagement im Unternehmen einzubinden. Denn das intelligente Energieversorgungssystem als systemrelevante kritische Infrastruktur stellt besonders hohe Anforderungen an Schutzmaßnahmen gegen Manipulationen und Angriffe - auch mit Blick auf die Zuverlässigkeit informationstechnischer Prozesse. Zunächst haben die Experten eine deutsche Spezifikation und schließlich darauf basierend einen internationalen Standard erstellt.

Deutschland hat damit gezeigt, dass es auch im Hinblick auf ITSicherheitsnormung einen Vorsprung durch Qualität und Schnelligkeit hat und Maßstäbe in der internationalen Normungspolitik setzen kann. Das Projekt TR 27019 steht modellhaft für Angebote zur Sicherstellung des technischen Schutzes für die „Digitale Gesellschaft" durch Standards und Normen. Dr. Ulrike Bohnsack, Mitglied der Geschäftsleitung des DIN, und Dr. Bernhard Thies, Sprecher der DKE-Geschäftsführung, würdigten in ihren Grußworten die gemeinsame Zusammenarbeit von DKE und DIN zur ISO/IEC TR 27019 als ein sehr positives Beispiel, wie im Interesse der deutschen Wirtschaft die notwendige Normungsarbeit gemeinsam geleistet werden kann. Die zunehmende Technikkonvergenz der verschiedensten Technikbereiche verspricht für die Zukunft eine steigende Zahl solcher gremienübergreifender Zusammenarbeiten

\section{Neue HSM-Serie von Utimaco: CryptoServer CSe - doppelte Leistung auf höchstem Sicherheitsniveau}

Utimaco stellt die neuste Generation ihrer FIPS 140-2 Level 4 zertifizierten Sicherheitsmodule vor. Die neue CSe-Serie trumpft insbesondere bei der Berechnung der elliptischen Kurven mit bis zu 1.500 Operationen pro Sekunde. Damit verfügt sie über die schnellste Software-Implementation am HSM Markt. „Wir haben für unsere Kunden immer die Gesamtbetriebskosten im Auge: So konnten wir die Anschaffungskosten auf dem Niveau des Vorgängermodells halten und die Betriebskosten sogar senken. Der Nachhaltigkeitsgedanke hat für uns seit jeher einen hohen Stellenwert", erläutert Matthias Pankert, VP HSM. Konkret steht der doppelten Crypto-Leistung nur ein unwesentlich gestiegener Stromverbrauch auf 6 statt vormals 5 Watt gegenüber. Dies ist auch unter ökologischen Gesichtspunkten interessant, denn bezogen auf die einzelne Crypto-Operation sinkt der effektive Stromverbrauch um gut 30 Prozent.

Ebenso wie ihr Vorgänger erfüllt auch die CSe-Serie als eins der wenigen HSMs am Markt die Anforderungen für die FIPS 140-2 Level 4 Zertifizierung für physikalische Sicherheit. Damit ist sie neben den klassischen Branchen besonders geeignet für Anwendungen in den hochsensiblen Wachstumsbranchen wie etwa Smart-Metering, elD oder eHealth.

Technisch gesehen verfügt die CSe-Serie neben der verbesserten Software-Implementation über einen PCI Express Bus zur schnelleren Kommunikation, einen neuen Prozessor, 2 USB-Anschlüsse sowie die 2 bis 8 -fache Speicherkapazität. Wie alle Utimaco HSMs ist die CSe-Serie sowohl als 19" Netzwerk-Appliance als auch als Einsteckkarte erhältlich.

„Wir sind auch dieses Mal unserer Philosophie gefolgt und haben bei der Entwicklung der CSe-Serie die Investitionssicherheit für unsere Kunden im Auge gehabt", erläutert Dieter Bong, Produktmanager CryptoServer. „Dank der Rückwärts-Kompatibilität unserer Architektur laufen Firmware-Anwendungen deshalb auch weiterhin auf der CSe-Serie und es ist sogar ein gemischter Betrieb von CS- und CSe-Serie im Verbund möglich." Auch die Tamper-Technologie bleibt mit Material und Verarbeitung auf dem gewohnten Niveau höchster Sicherheit. Zertifizierungen für FIPS 140-2 Level 3 und 4 sowie für die Deutsche Kreditwirtschaft laufen und werden zum Jahreswechsel erwartet.

\section{Trend Micro veröffentlicht juristischen IT-Leitfaden für Unternehmen}

Wie wichtig der Schutz von Unternehmensdaten und geistigem Eigentum im Zeitalter der Cyberspionage und -kriminalität ist, hat nicht zuletzt die aktuelle Diskussion um PRISM und Tempora vor Augen geführt. Zumal unzureichend abgesicherte IT-Infrastrukturen nicht nur Datenspionen Tür und Tor öffnen, sondern auch datenschutzrechtliche Konsequenzen nach sich ziehen. Was Unternehmen zu beachten haben, hat der IT-Sicherheitsanbieter Trend Micro in seinem juristischen Leitfaden zusammengefasst: Das in dritter, überarbeiteter Auflage im September 2013 erschienene Nachschlagewerk, das speziell auf die schweizerische Rechtsprechung sowie landesspezifische Vorschriften abgestimmt ist, gibt Unternehmensverantwortlichen Einblicke in juristische Themengebiete, die beim Einsatz von IT-Infrastruktur und Internet im Unternehmen relevant sind. Es steht als eBook zum kostenlosen Download bereit.

Welche Gefahren können aus der privaten E-Mail- und Internetnutzung durch die Mitarbeiter entstehen? Mit welchen konkreten Maßnahmen lässt sich unternehmensweite IT-Sicherheit umsetzen? Welchen Anforderungen müssen elektronische Dokumente und Rechnungen genügen, um rechtswirksam zu sein? Unter welchen Umständen werden sie als Beweismaterial vor Gericht anerkannt? Um rechtliche Konsequenzen für das eigene Unternehmen verhindern zu können, müssen Unternehmensverantwortliche sich mit solchen Fragen beschäftigen - und sie müssen über geltende Regelungen und landes- sowie branchenspezifische Vorschrif- 